\title{
INFINITESIMAL OPERATIONS ON COMPLEXES OF GRAPHS
}

\author{
JIM CONANT AND KAREN VOGTMANN
}

\begin{abstract}
In two seminal papers Kontsevich used a construction called graph homology as a bridge between certain infinite dimensional Lie algebras and various topological objects, including moduli spaces of curves, the group of outer automorphisms of a free group, and invariants of odd dimensional manifolds. In this paper, we show that Kontsevich's graph complexes, which include graph complexes studied earlier by Culler and Vogtmann and by Penner, have a rich algebraic structure. We define a Lie bracket and cobracket on graph complexes, and in fact show that they are Batalin-Vilkovisky algebras, and therefore Gerstenhaber algebras. We also find natural subcomplexes on which the bracket and cobracket are compatible as a Lie bialgebra.

Kontsevich's graph complex construction was generalized to the context of operads by Ginzburg and Kapranov, with later generalizations by GetzlerKapranov and Markl. In [CoV], we show that Kontsevich's results in fact extend to general cyclic operads. For some operads, including the examples associated to moduli space and outer automorphism groups of free groups, the subcomplex on which we have a Lie bi-algebra structure is quasi-isomorphic to the entire connected graph complex. In the present paper we show that all of the new algebraic operations canonically vanish when the homology functor is applied, and we expect that the resulting constraints will be useful in studying the homology of the mapping class group, finite type manifold invariants and the homology of $O u t\left(F_{n}\right)$.
\end{abstract}

\section{INTRODUCTION}

In [K1] and [K2, M. Kontsevich investigated three "worlds," or operads, which he called commutative, associative, and Lie. For each of these operads he defined an infinite dimensional symplectic Lie algebra and a chain complex of graphs, and then used invariant theory to prove that the graph complex computes the homology of the Lie algebra. Ginzburg and Kapranov generalized the notion of graph complex to the case of an arbitrary operad, calling the result the cobar complex [GiK]. Later E. Getzler and M. Kapranov GK2 generalized Kontsevich's graph complex construction to the class of differential graded modular operads, and called the resulting functor to graph complexes the Feynman transform. M. Markl [M] also gave a construction of graph complexes, in the context of cyclic operads. The purpose of the present paper is to demonstrate that these graph complexes, i.e. the image of the Getzler-Kapranov Feynman transform, carry a rich algebraic structure. In [Co, much of this structure is extended to give additional, higher-order algebraic operations.

1991 Mathematics Subject Classification. 17B62, 17B63, 17B70, 20F28, 57M07, 57M15, 57M27

The first author was partially supported by NSF VIGRE grant DMS-9983660. The second author was partially supported by NSF grant DMS-9307313. 
For the associative operad, Kontsevich's graph complex is the same as one defined by Penner $[\mathrm{P}$ to study moduli spaces of punctured surfaces, whereas in the Lie case, the graph complex comes from Culler and Vogtmann's "outer space," which they used to study the group of outer automorphisms of a free group (see $[\mathrm{CuV}]$ ). The commutative operad gives rise to what Kontsevich refers to as "graph homology." Homology classes which correspond to trivalent graphs parameterize finite type 3manifold invariants (see, e.g., [BGRT], [KT], [LMO]). The homology in other degrees parameterizes invariants of manifolds of higher odd dimension. In the commutative case, the associated Lie algebra $c_{\infty}$ can be identified with the direct limit of Lie algebras $c_{n}$, where $c_{n}$ is the Lie algebra of polynomial functions on $\mathbb{R}^{2 n}$ with no linear or constant terms, under the standard Poisson bracket. Alternatively, $c_{n}$ can be described as the Lie algebra of derivations of a polynomial algebra which preserve the symplectic form. The equivalence of these two descriptions comes from the correspondence, given by the symplectic form, between the Lie algebra of functions and the Lie algebra of vector fields on $\mathbb{R}^{2 n}$.

The commutative graph complex is spanned by oriented graphs, where the orientation can be most easily described as an equivalence class of certain labellings of edges and vertices. The chain complex is graded by the number of vertices in a graph, and the boundary operator $\partial_{E}$ is given by summing over all edge contractions. The appropriate notion of induced orientation guarantees that the square of $\partial_{E}$ is zero.

After examining Kontsevich's paper closely, we discovered the implicit presence of another boundary operator $\partial_{H}$, which anticommutes with $\partial_{E}$. It showed up as an error term in a certain diagram that needed to commute, and represented an oversight in Kontsevich's argument. In [CoV], we repair the gap in the more general context of cyclic operads.

This boundary operator is defined by contracting over pairs of half-edges. (See Figure 3 which depicts the contraction of the two half edges $h$ and $k$.) The commutative graph complex is a Hopf algebra, with multiplication given by disjoint union and comultiplication defined as $1 \otimes X+X \otimes 1$ on connected graphs and extended multiplicatively. It is easy to see that $\partial_{E}$ is both a derivation and a coderivation with respect to these operations. However, $\partial_{H}$ is neither of these. Instead, it satisfies the Batalin-Vilkovisky axiom, which implies that the defect from being a derivation is a Lie bracket. Similarly the deviation from being a coderivation is a Lie cobracket on graphs. We at first expected these operations to fit together as a Lie bialgebra, but it turns out they are only compatible on the subcomplex of connected graphs with no separating edges. In the Lie and associative cases this subcomplex carries the homology, as we show in [CoV]. Computer calculations of F. Gerlits Gts indicate that this is not so in the case we concentrate on in this paper, the commutative case. However, in CoGV we show that our operations induce a Lie bialgebra structure on an appropriate quotient complex which does indeed carry the homology.

These operations give a rich algebraic structure to the functor assigning chain complexes to cyclic operads. In the world of Lie algebras, there is a similar natural functor sending a Lie algebra $\mathfrak{g}$ to the exterior algebra $\Lambda \mathfrak{g}$, considered to be a chain complex with the Chevalley-Eilenberg differential. This is endowed with the Schouten bracket, which is also killed by applying the homology functor. In the last 
section of this paper we prove that our bracket comes from the Schouten bracket on the exterior algebra $\Lambda c_{\infty}$.

Sullivan and Chas have studied similar algebraic structures on the homology of free loop spaces. $(\underline{\mathrm{CS}}, \underline{\mathrm{C}})$ ). In particular they find a Batalin-Vilkovisky structure, a Lie bialgebra structure, and an uncountable infinity of Lie $e_{\infty}$ structures. These operations are generically present at the chain complex level but do not form a BV algebra until passing to homology.

The new algebraic operations described in this paper may be useful in studying the three topological applications of Kontsevich's theory: finite type invariants of odd dimensional manifolds, the homology of the mapping class group and the homology of $\operatorname{Out}\left(F_{n}\right)$. The vanishing of these operations homologically imposes serious constraints on these objects, and in particular may be useful in obtaining information about dimension.

We also hope that the new operations will be useful in the analysis of the Feynman transform functor, which Getzler and Kapranov show is a homotopy equivalence between certain categories of modular operads.

In this paper we study the properties of the new differential $\partial_{H}$ for the commutative graph complex. In $[\mathrm{Co}$ and $[\mathrm{CoV}]$ we explain the modifications of this paper which are necessary to define bracket and cobracket for general cyclic operads.

We would like to thank F. Gerlits, E. Getzler, S. Mahajan, D. Sullivan, and D. Thurston for their interest and stimulating conversations.

\section{Chain COMPlexes of GRAPhS}

In this section we describe Kontsevich's commutative graph complex $\mathcal{G}$ and the two boundary operators, $\partial_{E}$ and $\partial_{H}$.

By a graph we mean a finite 1-dimensional $C W$-complex $X$, with vertices $v(X)$ and edges $e(X)$. We assume that all vertices in the graph have valence at least 3 . An orientation on a graph $X$ is simply an orientation of the vector space $\mathbb{R}^{e(X)} \times$ $H_{1}(X ; \mathbb{R})$. We will usually find it more convenient to think of an orientation as an equivalence class of labellings, where a labelling of $X$ consists of an ordering of the vertices $v(X)$ and arrows on all edges. Reversing the arrow on any edge, or switching the order of two vertices changes the orientation. These two notions are equivalent for connected graphs (See $[\mathrm{T}],[\mathrm{KT}]$ for an explanation of this equivalence).

The $k$-chains of $\mathcal{G}$ are linear combinations of oriented graphs $(X$, or $)$ with $k$ vertices, modulo the relation $(X, o r)=-(X,-o r)$. This relation forces all graphs with loops to be zero, since one can switch the arrow on the loop to get an isomorphic graph with the opposite orientation, giving $(X, o r)=-(X$, or $)$. Therefore we may assume that our graphs have no loops.

Given an edge $e$ of $X$, we define $X_{e}$ to be the graph obtained from $X$ by collapsing $e$ to a point. The first boundary operator $\partial_{E}$ is given by summing over all possible edge collapses:

Definition 2.1. Let $(X$, or $)$ be an oriented graph. Then

$$
\partial_{E}(X, o r)=\sum\left(X_{e}, \text { or }\right)
$$

where the sum is over all edges $e$ of $X$, and $X_{e}$ is given the orientation induced from the orientation on $X$. 
To specify the induced orientation on $X_{e}$ in terms of labelled graphs, choose a representative for the orientation of $X$ such that the initial vertex of $e$ is labelled 1 and the terminal vertex is labelled 2. The labelling on $X_{e}$ is then given by the following rule: the vertex which results from collapsing $e$ is numbered 1 and the numbering on all other vertices is reduced by one. The arrows on all uncontracted edges are unchanged.

Lemma 2.2. Choose a labelling to represent the oriented graph $X$. Then collapsing an edge of $X$ from vertex $i$ to vertex $j$ with $i<j$ induces the orientation $(-1)^{j}$ or, where or is the orientation which results from numbering the collapsed edge $i$ and reducing the numbering on the vertices labelled $j, \ldots, n$ by 1.

Since all of our graphs will be oriented, we will suppress the orientation in our notation, writing simply $X$ instead of $(X, o r)$.

Since the boundary operator preserves the first Betti number, or loop degree of a graph, one can decompose graph homology as a direct sum over the homologies of a fixed loop degree. For loop degree two, there is only one possible graph, the theta graph. (All other possibilities are excluded because they have loops or vertices of valence 1 or 2.) Therefore the theta graph gives rise to a degree two homology class. In loop degree three, there are two possible graphs which have four vertices, as shown in Figure 1

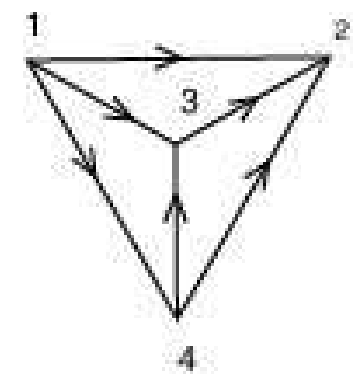

A
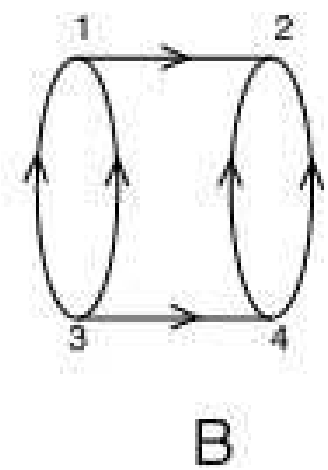

Figure 1. Graphs A and B

There is only one graph with three vertices and no loops, shown in Figure 2

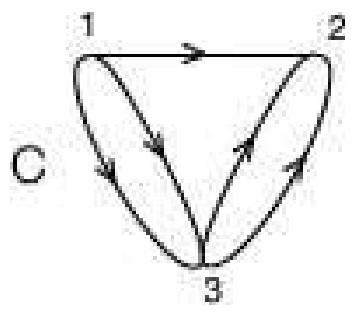

Figure 2. Graph C 
There is also a graph on two vertices with no loops, but this has an orientation reversing automorphism. It is easy to see that $\partial_{E} A=-6 C$ and $\partial_{E} B=2 C$. Hence we get one homology class in degree four, $A+2 B$.

To describe the second boundary operator on $\mathcal{G}$, we use the half-edges of a graph $X$. Each half-edge $h$ begins at a vertex $v(h)$, is contained in an edge $e(h)$ and has a complementary half-edge $\bar{h}$, with $h \cup \bar{h}=e(h)$. Given two half-edges $h$ and $k$ of $X$, we form a new graph $X\langle h k\rangle$ by cutting and pasting, as follows: if $k=\bar{h}$, then $X\langle h k\rangle=X$; if $k \neq \bar{h}$, we cut to separate $h$ from $\bar{h}$ and $k$ from $\bar{k}$, then glue $h$ to $k$ and $\bar{h}$ to $\bar{k}$ to form two new edges (see Figure [3). In terms of labelled graphs, the orientation on $X\langle h k\rangle$ is given as follows: choose a representative for the orientation on $X$ so that $h$ is the initial half-edge of $e(h)$, and $k$ is the terminal half-edge of $e(k)$. In $X\langle h k\rangle$, the edge $h \cup k$ is oriented from $h$ to $k$, and the edge $\bar{h} \cup \bar{k}$ is oriented from $\bar{k}$ to $\bar{h}$.
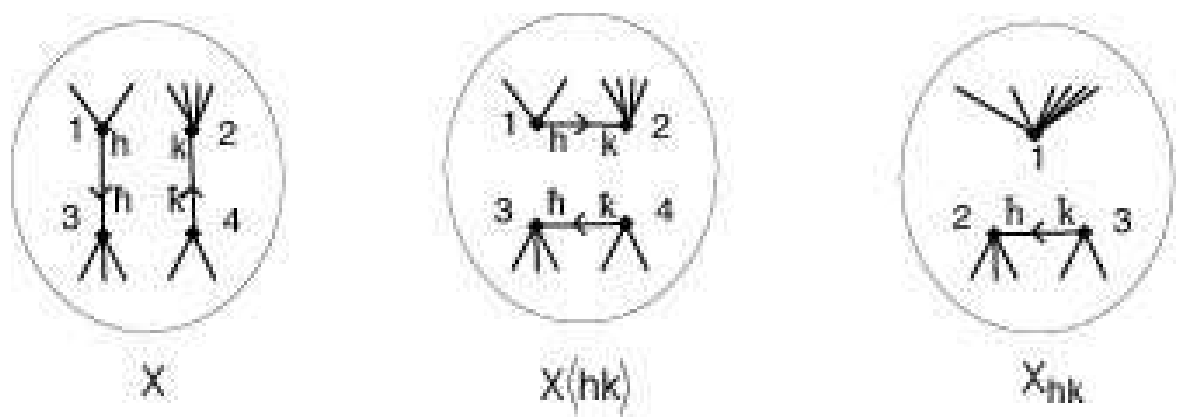

Figure 3. Contracting half-edges $h$ and $k$

If $h$ and $k$ are half-edges of $X$ with $v(h) \neq v(k)$, then $h \cup k$ forms an edge of $X\langle h k\rangle$, which we can now collapse; the result, $(X\langle h k\rangle)_{h \cup k}$, is more simply denoted $X_{h k}$. If, on the other, hand, $v(h)=v(k)$, then $X\langle h k\rangle$ has a loop, so is equal to 0; thus we define $X_{h k}$ to be 0 . Note that $X_{h k}=X_{k h}$ as oriented graphs.

The second boundary map on $\mathcal{G}$ is given by

\section{Definition 2.3.}

$$
\partial_{H} X=\sum X_{h k}
$$

where the sum is over all pairs $\{h, k\}$ of half-edges of $X$ with $h \neq \bar{k}$, and $X_{h k}$ is given the orientation induced from $X$.

To check that $\partial_{E}$ and $\partial_{H}$ are boundary operators, we use the following lemma:

Lemma 2.4. (Orientation Lemma) For any four distinct half-edges $h, k, r, s$ of $X$,

$$
\left(X_{h k}\right)_{r s}=-\left(X_{r s}\right)_{h k} \text {. }
$$

Proof. $\left(X_{h k}\right)_{r s}$ and $\left(X_{r s}\right)_{h k}$ are the results of collapsing the edges $h \cup k$ and $r \cup s$ of $X\langle h k\rangle\langle r s\rangle=X\langle r s\rangle\langle h k\rangle$, in the opposite order. Now observe that collapsing two edges of an oriented graph in opposite order results in isomorphic graphs with opposite orientations, using Lemma 2.2

Proposition 2.5. $\partial_{E}^{2}=\partial_{H}^{2}=\left(\partial_{E}+\partial_{H}\right)^{2}=0$ 
Proof. We have

$$
\left(\partial_{E}+\partial_{H}\right)^{2}(X)=\sum\left(X_{h k}\right)_{r s}
$$

where the sum is over all sets $\{h, k\}$ and $\{r, s\}$ of half-edges with $h, k, r$ and $s$ distinct. These terms cancel in pairs, by Lemma 2.4

Similarly, the terms in both squares $\left(\partial_{E}\right)^{2}(X)$ and $\left(\partial_{H}\right)^{2}(X)$ cancel in pairs: for $\partial_{E}$, the sum is over all sets $\{h, \bar{h}\}$ and $\{k, \bar{k}\}$ with $\{h, \bar{h}\} \neq\{k, \bar{k}\}$, and for $\partial_{H}$ the sum is over all sets of pairs $\{h, k\}$ and $\{r, s\}$ with $k \neq \bar{h}, s \neq \bar{r}$ and $\{r, s\} \neq\{\bar{h}, \bar{k}\}$ (equivalently, $\{h, k\} \neq\{\bar{r}, \bar{s}\}$ ).

Corollary 2.6. $\partial_{E} \partial_{H}=-\partial_{H} \partial_{E}$

We also briefly mention a slightly different, suggestive visualization of $\partial_{H}$. The terms of $\partial_{H} X$ naturally group themselves into sets of four, namely the four graphs $X_{h k}$ which can be formed from the half edges contained in a given pair of edges. We represent each such set of four graphs graphically by drawing a dotted line between the corresponding full edges (see Figure 4). Now $\partial_{H} X$ is given by summing over

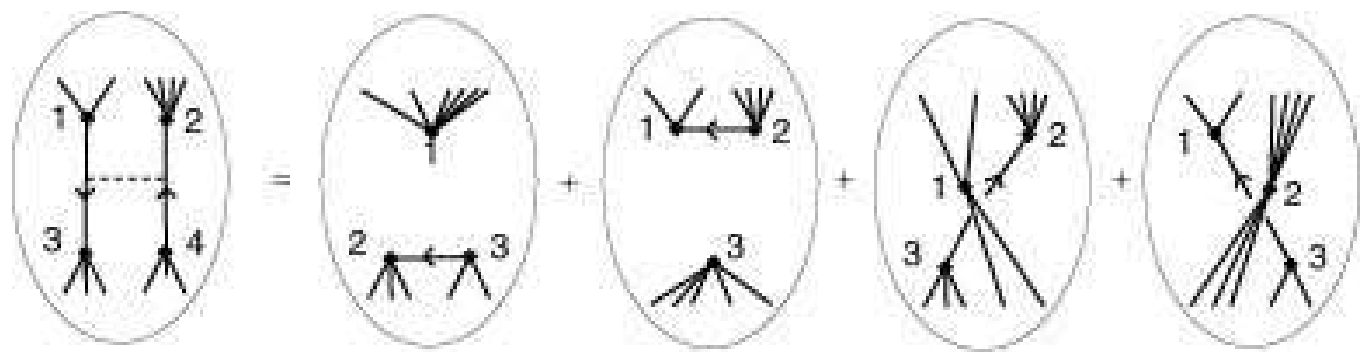

Figure 4. Dotted line notation

all possible ways of drawing a dotted line between two edges of $X$. The fact that $\partial_{H}^{2}=0$ can be derived from the two identities pictured in Figures 5 and [6] Figure 5 represents an antisymmetry relation, where the dotted lines are numbered to represent the order in which the operations are performed. Figure 6 represents a

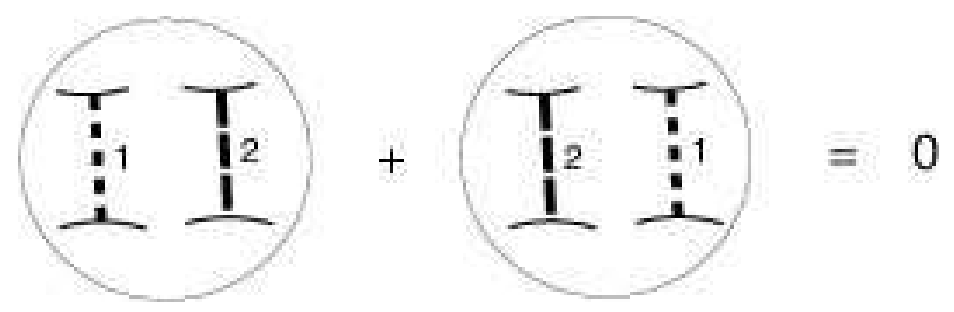

Figure 5. Antisymmetry

sort of Jacobi identity, where a second dotted line coming into a dotted line means that the second dotted line will attach to the uncontracted edge in each summand coming from the first dotted line. When we later define a bracket on graphs, these two identities can be used to give an alternative proof that this bracket is a Lie bracket. 


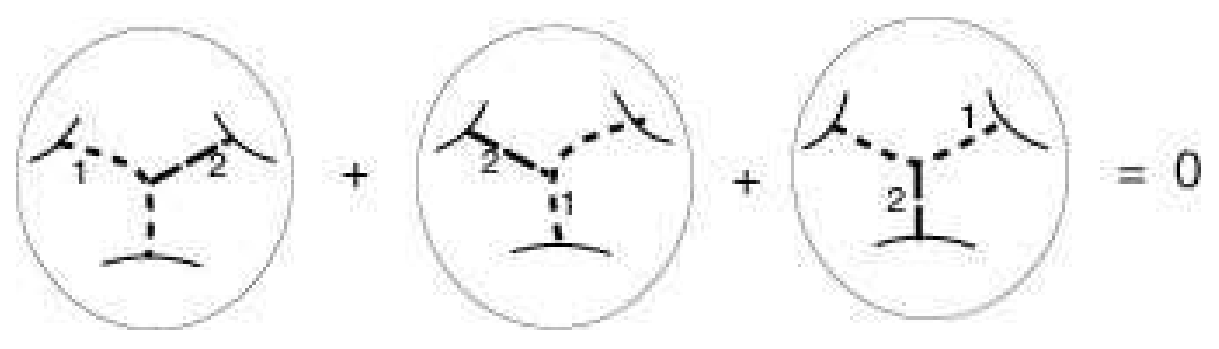

FiguRE 6. Jacobi relation

Kontsevich's "associative" and "Lie" complexes have similar descriptions to the above ("commutative") complex, except that the graphs have additional structure. In the associative case graphs come with a cyclic ordering of the edges incident to each vertex. In the Lie case an equivalence class of trivalent trees with $r$ leaves (modulo antisymmetry and IHX, or "Jacobi," relations) is associated to each vertex of valence $r$. The operations of cutting, pasting and collapsing described above can be done in such a way as to induce natural cyclic orderings or trees on the new vertices created, so that the boundary operators $\partial_{H}$ and $\partial_{E}$ have natural definitions in these settings as well. In this paper, we present only the commutative case, for simplicity; details of the remaining cases will appear in [CoV].

\section{Some GRAded ALGEBra}

Both chain complexes and their homology have the structure of graded vector spaces. We describe algebraic structures in this paper which are cognizant of the grading on the chain complexes of graphs, and descend to structures on homology. In this section we collect some standard definitions from graded algebra. We also record how things change under a grading shift.

Suppose $V$ is a graded vector space, either Z-graded $\left(V=\oplus_{n \geq 0} V_{n}\right)$ or $\mathbf{Z} / \mathbf{2}$ graded $\left(V=V_{0} \oplus V_{1}\right)$. We denote by $|v|$ the degree of $v \in V$. A linear map $T: V \rightarrow W$ of graded vector spaces is said to have degree $d$ if $|T(v)|=|v|+d$ for all homogeneous $v \in V$.

In order to make algebraic structures derived from graded vector spaces reflect the grading, we use the twist map $\tau: V \otimes W \rightarrow W \otimes V$, which takes $v \otimes w$ to $(-1)^{|v||w|} w \otimes v$. This extends to an isomorphism

$$
V_{1} \otimes V_{2} \cdots \otimes V_{k} \rightarrow V_{\pi(1)} \otimes V_{\pi(2)} \otimes \cdots \otimes V_{\pi(k)}
$$

for any permutation $\pi \in \Sigma_{k}$ and graded vector spaces $V_{1}, V_{2}, \ldots, V_{k}$; for example, the cyclic permutation (123) in $\Sigma_{3}$ gives an isomorphism $\sigma: V \otimes V \otimes V \rightarrow V \otimes V \otimes V$ defined by

$$
\sigma(u \otimes v \otimes w)=(-1)^{|w|(|u|+|v|)} w \otimes u \otimes v .
$$

In general, the sign is determined by "Koszul rule of signs": every time one switches two adjacent terms in a tensor product, the sign changes by the product of their degrees.

The isomorphisms given by the Koszul rule can be interpreted as new actions of $\Sigma_{k}$ on the tensor product of $k$ copies of a graded vector space $V$ : there is a symmetric action,

$$
\pi \cdot\left(v_{1} \otimes \cdots \otimes v_{k}\right)=(\text { Koszul sign }) v_{\pi(1)} \otimes \cdots \otimes v_{\pi(k)}
$$


and an alternating action,

$$
\pi \cdot\left(v_{1} \otimes \cdots \otimes v_{k}\right)=(\text { Koszul sign })(\operatorname{sign}(\pi)) v_{\pi(1)} \otimes \cdots \otimes v_{\pi(k)}
$$

Definition 3.1. The graded wedge product, denoted $\Lambda^{k} V$, is the quotient of $\otimes^{k} V$ by the alternating action of $\Sigma_{n}$, and the graded symmetric product, denoted $\odot^{k} V$ or $S^{k} V$, is the quotient of $\otimes^{k} V$ by the symmetric action of $\Sigma_{n} .{ }^{1}$

Let $V[-n]$ denote $V$ with the grading shifted downward by $n$. The wedge product and symmetric product are related as follows.

Proposition 3.2. There is a natural additive isomorphism $\phi: S^{*} V \rightarrow \Lambda^{*}(V[-1])$, defined inductively by

$$
\phi(A \odot B)=(-1)^{|A|} \phi(A) \wedge \phi(B),
$$

where $\left|v_{1} \odot \ldots \odot v_{k}\right|=\left|v_{1}\right|+\ldots+\left|v_{k}\right|$ and $\phi(v)=v$ for $v \in V$.

In particular, for $k=2$ this isomorphism is given by $v \odot w \mapsto(-1)^{|v|} v \wedge w$, and for $k=3$ by $u \odot v \odot w \mapsto(-1)^{|v|} u \wedge v \wedge w$.

The graded definition of Lie algebra $[\mathrm{M}-\mathrm{M}]$ is as follows.

Definition 3.3. A graded Lie bracket is a linear map $b: V \otimes V \rightarrow V$, written $b(x \otimes y)=[x, y]$, satisfying

- 1. (Graded antisymmetry)

$$
[v, w]=-(-1)^{|v||w|}[w, v]
$$

and

- 2. (Graded Jacobi identity)

$$
[u,[v, w]]+(-1)^{|w|(|u|+|v|)}[w,[u, v]]+(-1)^{|u|(|v|+|w|)}[v,[w, u]]=0
$$

An equivalent way to state the antisymmetry and Jacobi relations is that the following two compositions are zero:

- 1. $V \otimes V \stackrel{i d+\tau}{\longrightarrow} V \otimes V \stackrel{b}{\longrightarrow} V$

- 2. $V \otimes V \otimes V \stackrel{i d+\sigma+\sigma^{2}}{\longrightarrow} V \otimes V \otimes V \stackrel{i d \otimes b}{\longrightarrow} V \otimes V \stackrel{b}{\longrightarrow} V$.

This diagrammatic description is convenient because it allows us to define a graded Lie cobracket by simply reversing all the arrows:

Definition 3.4. A graded Lie cobracket is a linear map $\theta: V \rightarrow V \otimes V$ satisfying 1. (Graded co-antisymmetry) $(i d+\tau) \circ \theta=0$

2. (Graded co-Jacobi identity) $\left(i d+\sigma+\sigma^{2}\right) \circ(i d \otimes \theta) \circ \theta=0$

Notice that when $V$ is finite-dimensional, $(V, b)$ is a Lie algebra if and only if $\left(V^{*}, b^{*}\right)$ is a Lie coalgebra. If $V=\oplus V_{i}$ is a direct sum of finite-dimensional vector spaces, then $\left(\oplus V_{i}, \oplus b_{i}\right)$ is a Lie algebra if and only if $\left(\oplus V_{i}^{*}, \oplus b_{i}^{*}\right)$ is a Lie coalgebra. We write $V^{\dagger}=\oplus V_{i}^{*}$ and $b^{\dagger}=\oplus b_{i}^{*}$ to avoid confusion with $V^{*}$ and $b^{*}$.

The definitions of bracket and cobracket can be reformulated in the following nice way. Notice that the antisymmetry conditions imply that the bracket and cobracket induce maps

$$
b: V \wedge V \rightarrow V
$$

\footnotetext{
${ }^{1}$ In the literature $\Lambda V$ often means what we denote by $S V$.
} 
and

$$
\theta: V \rightarrow V \wedge V
$$

(In the case of the bracket, we are thinking of $V \wedge V$ as a quotient of $V \otimes V$, and in the cobracket case as a submodule of $V \otimes V$.) Further, these maps can be extended to the entire algebra $\Lambda^{*} V$ to itself. The map $b$ extending the bracket is a coderivation, and is just the usual Lie-algebra-homology boundary map. The map $\theta$ extends as a derivation.

Lemma 3.5. The Jacobi identity is equivalent to the assertion that $b^{2}=0$. The co-Jacobi identity is equivalent to the assertion that $\theta^{2}=0$.

By the lemma, the Jacobi identity is precisely what is needed to make the Chevalley-Eilenberg complex $\Lambda^{*} V$ of a graded Lie algebra $V$ into a chain complex. There is another standard operation on $\Lambda^{*} V$, the Schouten bracket, which usually appears in the context of Lie algebras of vector fields.

Definition 3.6. Let $V$ be a graded Lie algebra. Then the Schouten bracket on $\Lambda^{*} V$ is defined as follows:

$$
\begin{aligned}
& {\left[v_{1} \wedge \ldots \wedge v_{p}, w_{1} \wedge \ldots \wedge w_{q}\right]=} \\
& \quad \sum_{i, j}(-1)^{\epsilon}\left[v_{i}, w_{j}\right] \wedge v_{1} \wedge \ldots \wedge \hat{v}_{i} \wedge \ldots \wedge v_{p} \wedge w_{1} \wedge \ldots \wedge \hat{w}_{j} \wedge \ldots \wedge w_{q}
\end{aligned}
$$

where

$$
\begin{aligned}
\epsilon= & i+j+p+1+\text { Koszul } \\
=i+j+ & p+1+\left|v_{i}\right|\left(\left|v_{1}\right|+\ldots\left|v_{i-1}\right|\right) \\
& \quad+\left|w_{j}\right|\left(\left|v_{1}\right|+\ldots\left|\hat{v_{i}}\right|+\ldots\left|v_{p}\right|+\left|w_{1}\right|+\ldots\left|w_{j-1}\right|\right)
\end{aligned}
$$

Notice that $i+j+p+1$ is the sign of the permutation bringing $v_{i}, w_{j}$ to the front of the wedge product.

To express the compatibility of bracket and cobracket in the graded setting we first review the condition in the ungraded setting. One way of doing this is as follows $([\mathrm{M}]$ ),

$$
\theta[v, w]=a d_{v}(\theta(w))-a d_{w}(\theta(v))
$$

where the adjoint action is extended to the tensor product as a derivation: $a d_{v}\left(w_{1} \otimes\right.$ $\left.w_{2}\right)=\left[v, w_{1}\right] \otimes w_{2}+w_{1} \otimes\left[v, w_{2}\right]$. This is the same as the following condition:

$$
\theta[v, w]=\left(b \otimes i d+(i d \otimes b) \circ \tau_{12}\right)(i d \otimes \theta)(i d-\tau)(v \otimes w),
$$

where $\tau_{12}$ is the transposition swapping the first two tensor factors. Let $\theta(v)=$ $\sum v_{1} \otimes v_{2}$ and $\theta(w)=\sum w_{1} \otimes w_{2}$. Adding the Koszul signs and the degrees $|b|$ of the bracket and $|\theta|$ of the cobracket in the graded situation, the above condition becomes

$$
\begin{aligned}
\theta[v, w]= & \left(b \otimes i d+(i d \otimes b) \tau_{12}\right)\left((-1)^{|\theta||v|} v \otimes w_{1} \otimes w_{2}\right. \\
& \left.-(-1)^{|v||w|+|\theta||w|} w \otimes v_{1} \otimes v_{2}\right) \\
= & (-1)^{|\theta||v|}\left[v, w_{1}\right] \otimes w_{2}-(-1)^{|v||w|+|\theta||w|}\left[w, v_{1}\right] \otimes v_{2} \\
& +(-1)^{|\theta||v|+|v|\left|w_{1}\right|+|b|\left|w_{1}\right|} w_{1} \otimes\left[v, w_{2}\right] \\
& -(-1)^{|v||w|+|\theta||w|+|w|\left|v_{1}\right|+|b|\left|v_{1}\right|} v_{1} \otimes\left[w, v_{2}\right]
\end{aligned}
$$


Now, passing to wedge products,

$$
\begin{aligned}
\theta[v, w]= & (-1)^{|\theta||v|}\left[v, w_{1}\right] \wedge w_{2}-(-1)^{|v||w|+|\theta||w|}\left[w, v_{1}\right] \wedge v_{2} \\
& +(-1)^{|\theta||v|+|v|\left|w_{1}\right|+|b|\left|w_{1}\right|} w_{1} \wedge\left[v, w_{2}\right] \\
& -(-1)^{|v||w|+|\theta||w|+|w|\left|v_{1}\right|+|b|\left|v_{1}\right|} v_{1} \wedge\left[w, v_{2}\right] \\
= & (-1)^{|\theta||v|}\left[v, w_{1}\right] \wedge w_{2}-(-1)^{|v||w|+|\theta||w|}\left[w, v_{1}\right] \wedge v_{2} \\
& +(-1)^{|\theta||v|+|v|\left|w_{1}\right|+|b|\left|w_{1}\right|+\left|w_{1}\right|\left(|v|+\left|w_{2}\right|+|b|\right)+1}\left[v, w_{2}\right] \wedge w_{1} \\
& -(-1)^{|v||w|+|\theta||w|+|w|\left|v_{1}\right|+|b|\left|v_{1}\right|+\left|v_{1}\right|\left(|w|+\left|v_{2}\right|+|b|\right)+1}\left[w, v_{2}\right] \wedge v_{1} \\
= & (-1)^{|\theta||v|}\left(\left[v, w_{1}\right] \wedge w_{2}+(-1)^{\left|w_{1}\right|\left|w_{2}\right|+1}\left[v, w_{2}\right] \wedge w_{1}\right) \\
& +(-1)^{|v||w|+|\theta||w|}\left((-1)^{\left|v_{1}\right||w|}\left[v_{1}, w\right] \wedge v_{2}\right. \\
& \left.+(-1)^{\left|v_{1}\right|\left|v_{2}\right|+\left|v_{2}\right||w|+1}\left[v_{2}, w\right] \wedge v_{1}\right) \\
= & (-1)^{|\theta||v|}\left[v, w_{1} \wedge w_{2}\right]+(-1)^{\left|v_{2}\right||w|}\left[v_{1}, w\right] \wedge v_{2} \\
& +(-1)^{\left|v_{1}\right|\left|v_{2}\right|+\left|v_{1}\right||w|+1}\left[v_{2}, w\right] \wedge v_{1} \\
= & (-1)^{|\theta||v|}\left[v, w_{1} \wedge w_{2}\right]-\left[v_{1} \wedge v_{2}, w\right]
\end{aligned}
$$

We therefore adopt the following definition of graded Lie bialgebra.

Definition 3.7. A graded Lie bialgebra is a graded vector space $V$ together with a Lie bracket $b=[\cdot, \cdot]: \Lambda^{2} V \rightarrow V$ and a Lie cobracket $\theta: V \rightarrow \Lambda^{2} V$ which are compatible:

$$
\theta[v, w]=-[\theta(v), w]+(-1)^{|v||\theta|}[v, \theta(w)],
$$

where the bracket on the right hand side of the equation is the Schouten bracket.

We would like to analyze now what happen when the degree is shifted. Recall (Proposition 3.2) that there is a natural isomorphism $\phi: \Lambda V[-1] \cong S V$. If one has an operation $b=[\cdot, \cdot]: S^{2} V \rightarrow V$, one gets an induced operation $b^{\phi}=$ $[\cdot, \cdot]^{\phi}: \Lambda^{2} V[-1] \rightarrow V$ defined by $b^{\phi}=b \circ \phi^{-1}$. Similarly, an operation $\theta: V \rightarrow S^{2} V$ gives rise to a map $\theta^{\phi}=\phi \circ \theta$.

Proposition 3.8. A linear map $b=[\cdot, \cdot]: S^{2} V \rightarrow V$ induces a graded Lie bracket $[\cdot, \cdot]^{\phi}$ on $V[-1]$ iff either of the following two equivalent conditions holds:

- 1. The extension of $b$ to $S V$ as a coderivation has trivial square.

- 2. (Graded Jacobi identity)

$$
[u,[v, w]]+(-1)^{|w|(|u|+|v|)}[w,[u, v]]+(-1)^{|u|(|v|+|w|)}[v,[w, u]]=0
$$

Note that the second axiom is the usual graded Jacobi identity!

Proposition 3.9. A linear map $\theta: V \rightarrow S^{2} V$ induces a graded Lie cobracket $\theta^{\phi}$ on $V[-1]$ iff the extension of $\theta$ to $S V$ as a derivation has trivial square.

As before, if $V=\oplus V_{i}$ is a direct sum of finite dimensional vector spaces we have that $\theta: V \rightarrow S^{2} V$ satisfies the graded co-Jacobi identity iff $\theta^{\dagger}$ satisfies the graded Jacobi identity. In othr words, $\theta^{\phi}$ is a Lie cobracket iff $\left(\theta^{\dagger}\right)^{\phi}$ is a Lie bracket.

In order to define the compatibility conditions between bracket and cobracket in the symmetric world, we need to transfer the Schouten bracket to the symmetric setting. 
Definition 3.10. Let $V$ be a graded Lie algebra. The symmetric Schouten bracket on $S V$ is defined by:

$$
\begin{aligned}
& {\left[v_{1} \odot \ldots \odot v_{p}, w_{1} \odot \ldots \odot w_{q}\right]=} \\
& \quad \sum_{i, j}(-1)^{\epsilon}\left[v_{i}, w_{j}\right] \odot v_{1} \odot \ldots \odot \hat{v}_{i} \odot \ldots \odot v_{p} \odot w_{1} \odot \ldots \odot \hat{w}_{c} \odot \ldots \odot w_{q}
\end{aligned}
$$

where

$$
\begin{aligned}
\epsilon & =\text { Koszul } \\
& =\left|v_{i}\right|\left(\left|v_{1}\right|+\ldots\left|v_{i-1}\right|\right)+\left|w_{j}\right|\left(\left|v_{1}\right|+\ldots\left|\hat{v_{i}}\right|+\ldots\left|v_{p}\right|+\left|w_{1}\right|+\ldots\left|w_{j-1}\right|\right) .
\end{aligned}
$$

Proposition 3.11. The linear maps $b: S^{2} V \rightarrow V$ and $\theta: V \rightarrow S^{2} V$ induce a graded Lie bialgebra structure on $V[-1]$ iff the following three conditions hold:

1. $b^{\phi}$ is a Lie bracket.

2. $\theta^{\phi}$ is a Lie cobracket.

3. $\theta([v, w])=-[\theta(v), w]-(-1)^{|v||\theta|}[v, \theta(w)]$

\section{Product AND BRACKeT}

Let $X$ be a labelled graph with vertices numbered $1,2, \ldots, x$, and $Y$ a labelled graph with vertices numbered $1,2, \ldots, y$. Define the product $X \cdot Y$ to be the disjoint union of $X$ and $Y$, with the numbering on vertices of $Y$ shifted by adding $x$ to each, thus becoming $x+1, \ldots, x+y$. Then we have

$$
X \cdot Y=(-1)^{x y} Y \cdot X .
$$

This product extends bilinearly to linear combinations of graphs, turning $\mathcal{G}$ into a graded commutative algebra. One may allow the empty graph as a basis element of $\mathcal{G}$, since it acts as a unit under the disjoint union operation.

Lemma 4.1. With respect to this product the boundary operator $\partial_{E}$ is a graded derivation:

$$
\partial_{E}(X \cdot Y)=\partial_{E}(X) \cdot Y+(-1)^{x} X \cdot \partial_{E}(Y) .
$$

Proof. This follows since each term $(X \cdot Y)_{e}$ of $\partial_{E}(X \cdot Y)$ is obtained by collapsing an edge $e$, which is either in $X$ or is in $Y$. The sign comes from the fact that if $e$ is an edge of $Y$, then $(X Y)_{e}=(-1)^{x} X Y_{e}$.

The second boundary operator $\partial_{H}$, on the other hand, is not a derivation; if $h \subset X$ and $k \subset Y$, then the term $(X \cdot Y)_{h k}$ of $\partial_{H}(X \cdot Y)$ is not in $\partial_{H}(X) \cdot Y+$ $(-1)^{x} X \cdot \partial_{H}(Y)$. We define the bracket $[X, Y]$ so that it measures how far $\partial_{H}$ is from being a derivation:

Definition 4.2. $[X, Y]=\partial_{H}(X \cdot Y)-\partial_{H}(X) \cdot Y-(-1)^{x} X \cdot \partial_{H}(Y)$.

In other words, the bracket of $X$ and $Y$ is the sum of all graphs obtained by contracting a half-edge of $X$ with a half-edge of $Y$ :

$$
[X, Y]=\sum_{h \in X, k \in Y}(X \cdot Y)_{h k}
$$

The bracket obeys symmetry and Jacobi relations as given in the following two lemmas: 
Lemma 4.3. Let $X$ and $Y$ be graphs with $x$ and $y$ vertices, respectively. Then $[X, Y]=(-1)^{x y}[Y, X]$.

Proof.

$$
\begin{aligned}
{[X, Y] } & =\sum_{h \in X, k \in Y}(X \cdot Y)_{h k} \\
& =(-1)^{x y} \sum_{h \in X, k \in Y}(Y \cdot X)_{h k} \\
& =(-1)^{x y}[Y, X] .
\end{aligned}
$$

Lemma 4.4. Let $X, Y$ and $Z$ be graphs with $x, y$, and $z$ vertices, respectively. Then

$$
[[X, Y], Z]+(-1)^{z(x+y)}[[Z, X], Y]+(-1)^{y(z+x)}[[Y, Z], X]=0 .
$$

Proof. We have

$$
\begin{aligned}
{[[X, Y], Z] } & =\sum\left((X \cdot Y)_{h k} \cdot Z\right)_{r s} \\
& =\sum\left((X \cdot Y \cdot Z)_{h k}\right)_{r s}
\end{aligned}
$$

where $h \in X, k \in Y, s \in Z$ and $r \in X \cup Y, r \notin\{h, k\}$.

If $r \in Y$, then by Lemma $2.4\left((X \cdot Y \cdot Z)_{h k}\right)_{r s}$ cancels with the term

$$
\left((X \cdot Y \cdot Z)_{r s}\right)_{h k}=(-1)^{z(x+y)}\left((X \cdot Y \cdot Z)_{r s}\right)_{h k},
$$

which is in $(-1)^{z(x+y)}[[Y, Z], X]$.

If $r \in X$ then $\left((X \cdot Y \cdot Z)_{h k}\right)_{r s}$ cancels with the term $\left((X \cdot Y \cdot Z)_{r s}\right)_{h k}$ of $(-1)^{x(y+z)}[[Z, X], Y]$.

The remaining terms of $(-1)^{x(y+z)}[[Z, X], Y]$ and $(-1)^{z(x+y)}[[Y, Z], X]$ similarly cancel in pairs.

The above two lemmas combine with Proposition 3.8 to give

Proposition 4.5. $\mathcal{G}[-1]$ is a graded Lie algebra with bracket $[\cdot, \cdot]^{\phi}$.

Remark. In terms of the dotted line notation we introduced after the definition of $\partial_{H}$, the bracket $[X, Y]$ is the sum over all possible ways of drawing a dotted line between an edge of $X$ and an edge of $Y$. An alternate proof of the fact that the bracket satisfies the Jacobi identity can be given using the antisymmetry and Jacobi identities of dotted lines.

In fact, the bracket gives a stronger structure on $\mathcal{G}[-1]$. Recall that a Gerstenhaber algebra (or graded Poisson algebra) is a graded commutative, associative algebra $V$ with a degree -1 Lie bracket, satisfying

$$
[u, v w]=[u, v] w+(-1)^{|u|(|v|+1)} v[u, w]
$$

Proposition 4.6. $\mathcal{G}[-1]$ is a Gerstenhaber algebra, under the product and bracket defined above. 
Proof. Let $X, Y$ and $Z$ be graphs with $x, y$ and $z$ vertices, respectively. Recall that the bracket on the shifted graph complex is denoted $[\cdot, \cdot]^{\phi}$. Then $[A, B]^{\phi}=$ $(-1)^{a}[A, B]$, where $a$ is the number of vertices of $\mathrm{A}$. Now

$$
\begin{aligned}
{[X, Y Z] } & =\sum_{h \subset X, k \subset Y Z}\left((X \cdot(Y \cdot Z))_{h k}\right. \\
& =\sum_{h \subset X, k \subset Y}(X \cdot Y \cdot Z)_{h k}+\sum_{h \subset G, k \subset Z}(X \cdot Y \cdot Z)_{h k} \\
& =\sum(X \cdot Y)_{h k} \cdot Z+(-1)^{z y} \sum(X \cdot Z)_{h k} \cdot Y \\
& =[X, Y] Z+(-1)^{z y}[X, Z] Y \\
& =[X, Y] Z+(-1)^{y(x-1)} Y[X, Z]
\end{aligned}
$$

Multiplying through by $(-1)^{x}$, and noticing that $|x|=x+1$ and $|y|=y+1$, we see that $[X, Y Z]^{\phi}=[X, Y]^{\phi} Z+(-1)^{|x|(|y|+1)}+Y[X, Z]^{\phi}$, as desired.

An algebraic structure that has recently gained attention is a Batalin-Vilkovisky algebra ([Ge,,$\underline{\mathrm{CS}})$. It is a graded commutative algebra together with with a degree 1 map $\Delta$ satisfying $\Delta^{2}=0$ and such that $(-1)^{|v|} \Delta(v w)-(-1)^{|v|}(\Delta v) w-v \Delta w$ is a derivation of each variable. That is,

$$
\begin{aligned}
\Delta(u v w)= & \Delta(u v) w+(-1)^{|u|} u \Delta(v w)+(-1)^{(|u|-1)|v|} v \Delta(u) \\
& -(\Delta u) v w-(-1)^{|u|} u(\Delta v) w-(-1)^{|u|+|v|} u v(\Delta w) .
\end{aligned}
$$

If we consider $\mathcal{G}$ as a $\mathbf{Z} / 2$-graded vector space, then $\partial_{H}$, which is a degree -1 operator, becomes "degree 1," and makes $\mathcal{G}$ into a super Batalin-Vilkovisky algebra.

Proposition 4.7. As a super algebra, $\mathcal{G}$ is a Batalin-Vilkovisky algebra with respect to the operator $\Delta=\partial_{H}$.

Proof. We know that $\partial_{H}^{2}=0$, so it suffices to check $\partial_{H}(X Y Z)$ is of the required form. This follows essentially because each term of $\partial_{H}$ can only affect at most two of $\{X, Y, Z\}$.

This provides an alternate proof that $(-1)^{x}[X, Y]$ is a graded Lie bracket because of the following proposition. (See $\mathrm{Ge}$, [C].)

Proposition 4.8. Any BV algebra is also a Gerstenhaber algebra (in the super sense), by defining the Lie bracket to satisfy:

$$
(-1)^{|v|}[v, w]=\Delta(v w)-\Delta(v) w-(-1)^{|v|} v \Delta(w) .
$$

\section{COproduct AND COBRACKET}

In addition to the product structure $\mu: \mathcal{G} \otimes \mathcal{G} \rightarrow \mathcal{G}$ on the graded vector space $\mathcal{G}$ of graphs, there is also a coproduct structure $\Delta: \mathcal{G} \rightarrow \mathcal{G} \otimes \mathcal{G}$. To describe this, note that the algebra structure on $\mathcal{G}$ induces an algebra structure on $\mathcal{G} \otimes \mathcal{G}$, by

$$
(X \otimes Y)(Z \otimes W)=(-1)^{y w} X W \otimes Y Z .
$$

For a connected graph $X$, we define $\Delta(X)=X \otimes 1+1 \otimes X$, where 1 denotes the empty graph. $\Delta$ is extended multiplicatively to all of $\mathcal{G}$ by $\Delta(X \cdot Y)=\Delta(X) \cdot \Delta(Y)$. For example, if $X$ and $Y$ are connected, then

$$
\Delta(X \cdot Y)=X \cdot Y \otimes 1+1 \otimes X \cdot Y+X \otimes Y+(-1)^{x y} Y \otimes X .
$$


As usual, it is convenient to express things diagramatically when defining "co"objects. If we extend a map $d: \mathcal{G} \rightarrow \mathcal{G}$ to $d: \mathcal{G} \otimes \mathcal{G} \rightarrow \mathcal{G} \otimes \mathcal{G}$ by the formula $d(X \otimes Y)=d(X) \otimes Y+(-1)^{x} X \otimes d(Y)$, the fact that $d$ is a derivation can be said efficiently as $d \mu=\mu d$, i.e. the following diagram commutes:

$$
\begin{array}{cccc}
\mathcal{G} \otimes \mathcal{G} & \stackrel{\mu}{\rightarrow} & \mathcal{G} \\
d \downarrow & & \downarrow d \\
\mathcal{G} \otimes \mathcal{G} & \stackrel{\mu}{\rightarrow} & \mathcal{G}
\end{array}
$$

To define a coderivation, we simply reverse the arrows and replace multiplication by comultiplication: a map $\delta$ is said to be a co-derivation if $\Delta \delta=\delta \Delta$, where $\delta$ is extended to $\mathcal{G} \otimes \mathcal{G}$ as before by $\delta(X \otimes Y)=\delta(X) \otimes Y+(-1)^{x} X \otimes \delta(Y)$ :

$\begin{array}{ccc}\mathcal{G} \otimes \mathcal{G} & \stackrel{\mathcal{G}}{\rightleftarrows} \\ \delta \uparrow & & \uparrow \delta \\ \mathcal{G} \otimes \mathcal{G} & \rightleftarrows & \mathcal{G}\end{array}$

Proposition 5.1. $\partial_{E}$ is a coderivation.

Proof. If $X$ is connected, then all terms of $\partial_{E}(X)=\sum_{e} X_{e}$ are connected, so that

$$
\begin{aligned}
\Delta\left(\partial_{E} X\right) & =\Delta\left(\sum_{e} X_{e}\right) \\
& =\sum_{e}\left(X_{e} \otimes 1+1 \otimes X_{e}\right) \\
& =\partial_{E} X \otimes 1+1 \otimes \partial_{E} X \\
& =\partial_{E}(X \otimes 1+1 \otimes X) \\
& =\partial_{E} \Delta(X)
\end{aligned}
$$

The case when $X$ is not connected can be handled as follows. Since $\partial_{E}$ is a derivation of $\mathcal{G}$, it is a derivation of $\mathcal{G} \otimes \mathcal{G}$. That is, for any $a, b, c$ and $d$ in $\mathcal{G}$,

$$
\partial_{E}(a \otimes b)(c \otimes d)=\left(\partial_{E}(a \otimes b)\right)(c \otimes d)+(-1)^{|a|+|b|}(a \otimes b) \partial_{E}(c \otimes d) .
$$

Apply this formula to $\partial_{E} \Delta(X Y)=\partial_{E}(\Delta(X) \Delta(Y))$, using that you inductively know $\partial_{E} \Delta(X)=\Delta \partial_{E}(X)$ and $\partial_{E} \Delta(Y)=\Delta \partial_{E}(Y)$.

When we try the same computation with $\partial_{H}$, we run into problems because the terms $X_{h k}$ in $\partial_{H}(X)$ may not be connected, even when $X$ is connected, and the first line of the calculation in the proof of Proposition[5.1 is not valid. For example, in Figure [7 contracting the half-edges $h$ and $k$ separates the graph into two pieces.

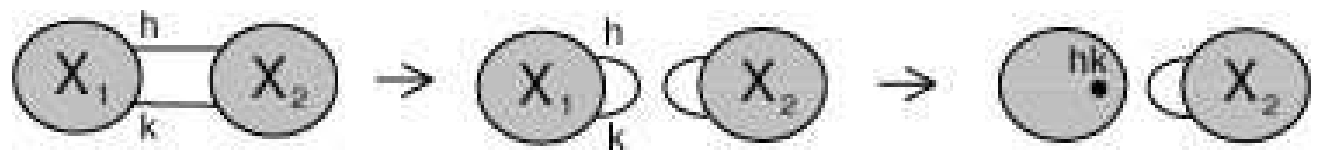

FiguRE 7. Separating pair of half-edges

By analogy with our definition of the bracket, we define the cobracket $\theta: \mathcal{G} \rightarrow$ $\mathcal{G} \otimes \mathcal{G}$ to measure how far $\partial_{H}$ is from being a coderivation, i.e. 
Definition 5.2. For any graph $X$, the cobracket $\theta(X)$ is defined to be

$$
\theta(X)=\Delta\left(\partial_{H} X\right)-\partial_{H} \Delta(X) .
$$

In terms of graphs, the cobracket has the following interpretation. If $X$ is connected, we say that a pair $\{h, k\}$ of half-edges separates $X$ if $X_{h k}$ is not connected. If $\{h, k\}$ separates a connected graph $X$, then $h$ and $k$ must be in one of the configurations depicted in Figure 8 where the graphs $X_{i}$ are connected. We compute
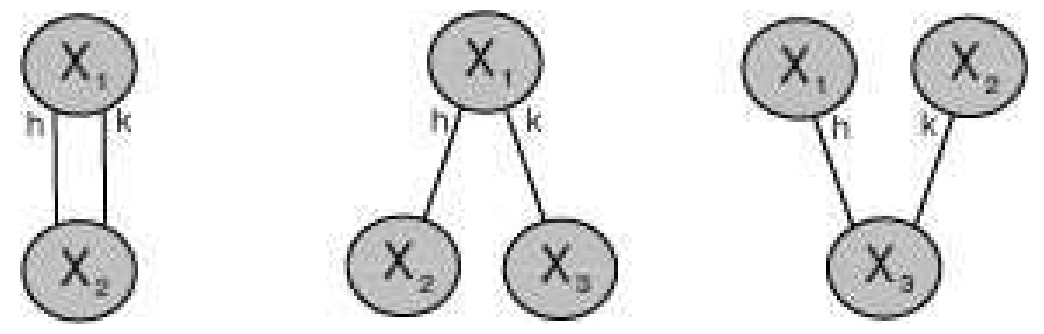

FiguRE 8. All configurations of a separating pair

$$
\begin{aligned}
\Delta\left(\partial_{H} X\right) & =\sum_{\{h, k\} \mid k \neq \bar{h}} \Delta\left(X_{h k}\right) \\
& =\sum_{X_{h k} \text { connected }}\left(X_{h k} \otimes 1+1 \otimes X_{h k}\right)+\sum_{X_{h k}=A_{h k} \cdot B_{h k}} \Delta\left(A_{h k} \cdot B_{h k}\right)
\end{aligned}
$$

In the second summand, note that the graphs $A_{h k}$ and $B_{h k}$ are connected. If $A_{h k}$ has $a$ vertices and $B_{h k}$ has $b$ vertices, we have

$$
\begin{aligned}
\Delta\left(A_{h k} \cdot B_{h k}\right)=\Delta\left(B_{h k} \cdot A_{h k}\right)=A_{h k} \cdot & B_{h k} \otimes 1+1 \otimes A_{h k} \cdot B_{h k} \\
& +A_{h k} \otimes B_{h k}+(-1)^{a b} B_{h k} \otimes A_{h k}
\end{aligned}
$$

On the other hand,

$$
\begin{aligned}
\partial_{H} \Delta(X) & =\partial_{H}(X \otimes 1+1 \otimes X) \\
& =\partial_{H} X \otimes 1+1 \otimes \partial_{H} X \\
& =\sum_{\{h, k\} \mid k \neq \bar{h}}\left(X_{h k} \otimes 1+1 \otimes X_{h k}\right)
\end{aligned}
$$

Thus the difference $\theta(X)$ between $\partial_{H} \Delta(X)$ and $\Delta\left(\partial_{H} X\right)$ is the sum, over all pairs $\{h, k\}$ of half-edges which separate $X$, of $A_{h k} \otimes B_{h k}+(-1)^{a b} B_{h k} \otimes A_{h k}$. We can simplify the notation by writing this in the symmetric algebra, as

$$
\theta(X)=\sum_{\{h, k\} \mid X_{h k}=A_{h k} \cdot B_{h k}} A_{h k} \odot B_{h k} .
$$

If $X$ is not connected, the formula is more complicated. Specifically, if $X=$ $X_{1} \cdot X_{2} \ldots \cdot X_{k}$, with all $X_{i}$ connected, we need to consider separating pairs $\{r, s\}$ in $X_{i}$ and pairs $\{r, s\}$ with $r$ separating in $X_{i}$ and $s$ separating in $X_{j}$ (see Figure 9). Given such a pair, write $\left(X_{i}\right)_{r s}=A \cdot B$ or $\left(X_{i} \cdot X_{j}\right)_{r s}=A \cdot B$, and list all 

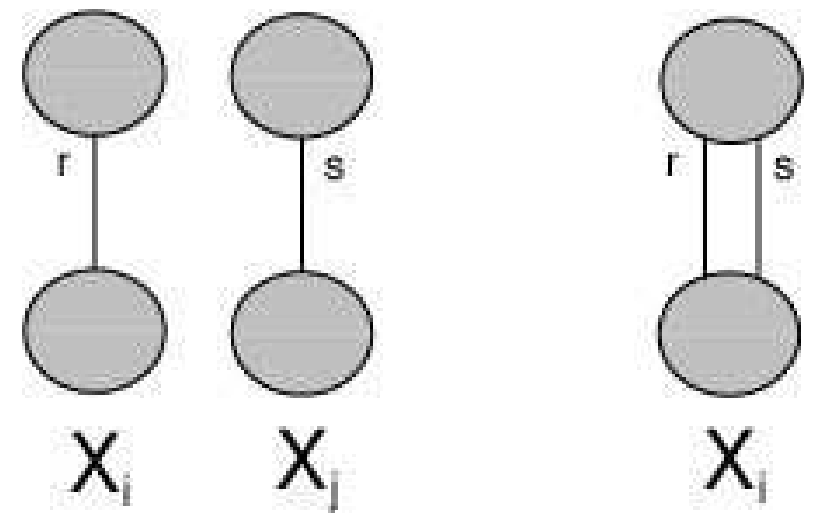

FiguRE 9. Separating pairs in disconnected graphs

ordered partitions $(I, J)$ of $\{1, \ldots, \hat{i}, \ldots, \hat{j}, \ldots, k\}$ into two subsets (either or both of which may be empty). Then

$$
\theta\left(X_{1} \cdot X_{2} \ldots \cdot X_{k}\right)=\sum_{\{r, s\}} \sum_{(I, J)}(-1)^{\kappa} X_{I} \cdot A \odot B \cdot X_{J},
$$

where $X_{I}$ is the product of the $X_{i}$ with $i \in I, X_{J}$ is the product of the $X_{j}$ with $j \in J$, and $\kappa$ is the Koszul sign. For example, if $k=2$, with $X_{1}=X$ and $X_{2}=Y$, then

$$
\begin{aligned}
\theta(X \cdot Y)= & \sum_{r \in X, s \in Y,(X \cdot Y)_{r s}=A \cdot B} A \odot B \\
& +\sum_{r, s \in X, X_{r s}=A \cdot B} A \odot B \cdot Y+(-1)^{a b} B \odot A \cdot Y \\
& +\sum_{r, s \in Y, Y_{r s}=A \cdot B} X \cdot A \odot B+(-1)^{a b} X \cdot B \odot A
\end{aligned}
$$

If we assume our graphs have no separating edges, then the first summand above vanishes, and the formula takes the following more elegant form:

$$
\theta(X \cdot Y)=\theta(X) \Delta(Y)+(-1)^{x y} \theta(Y) \Delta(X),
$$

where $X$ has $x$ vertices and $Y$ has $y$ vertices. In fact this formula holds for arbitrary graphs $X$ and $Y$, not necessarily connected. Here we have used the fact that $\mathcal{G}$ is graded cocommutative, hence the coproduct induces a map $\mathcal{G} \rightarrow \mathcal{G} \odot \mathcal{G}$.

\section{Proposition 5.3.}

is a graded Lie cobracket.

$$
\theta^{\phi}: \mathcal{G}[-1] \rightarrow \Lambda^{2} \mathcal{G}[-1]
$$

For the purposes of the proof it is easier to dualize. Since $\mathcal{G}$ is the direct sum $\oplus \mathcal{G}_{v, e}$ of vector spaces spanned by graphs with $v$ vertices and $e$ edges, and each $\mathcal{G}_{v, e}$ is finite dimensional, from our earlier remarks it follows that an operation $\theta: \mathcal{G} \rightarrow S^{2} \mathcal{G}$ induces a a graded Lie cobracket $\theta^{\phi}$ if and only if $\theta^{\dagger}=\oplus \theta_{v, e}^{*}$ satisfies 
the graded Jacobi identity on $\mathcal{G}^{\dagger}=\oplus \mathcal{G}_{v, e}^{*}$. Suppose $X \in \mathcal{G}$. Let $\langle X, \cdot>$ denote the unique functional such that, for any graph $Y$

$$
<X, Y>= \begin{cases}|A u t(X)|, & \text { if } X \cong Y \\ 0 & \text { otherwise }\end{cases}
$$

We denote the resulting isomorphism by $A: \mathcal{G} \rightarrow \oplus \mathcal{G}^{\dagger}$.

The boundary operator $\partial_{H}$ will be replaced in the dual setting by a coboundary operator $\delta_{H}: \mathcal{G}_{v} \rightarrow \mathcal{G}_{v+1}$, defined as follows:

Let $X$ be a graph, and let $(P, \bar{P})$ be a partition of the edges incident to a vertex $v$ of $X$. Expand the vertex $v$ to obtain a new graph $X^{(P, \bar{P})}$, with a new edge separating $P$ from $\bar{P}$. This new edge is the union of two half-edges, which we name $p$ and $\bar{p}$ to reflect the original edges of $X$ to which they are now incident. The orientation on $X^{(P, \bar{P})}$ is chosen so that collapsing the new edge gives back the original orientation on $X$. Given a half-edge $h$ in $X$, we can now form the graph $X^{(P, \bar{P})}\langle p h\rangle$ for the graph obtained from $X^{(P, \bar{P})}$ by cutting and pasting together the two half-edges $p$ and $h$. If both $P$ and $\bar{P}$ have at least two elements, we denote this new graph by $X^{P h}$; otherwise we set $X^{P h}=0$ (see Figure 10).

Lemma 5.4. As oriented graphs, $X^{P h}=X^{\bar{P} \bar{h}}$.
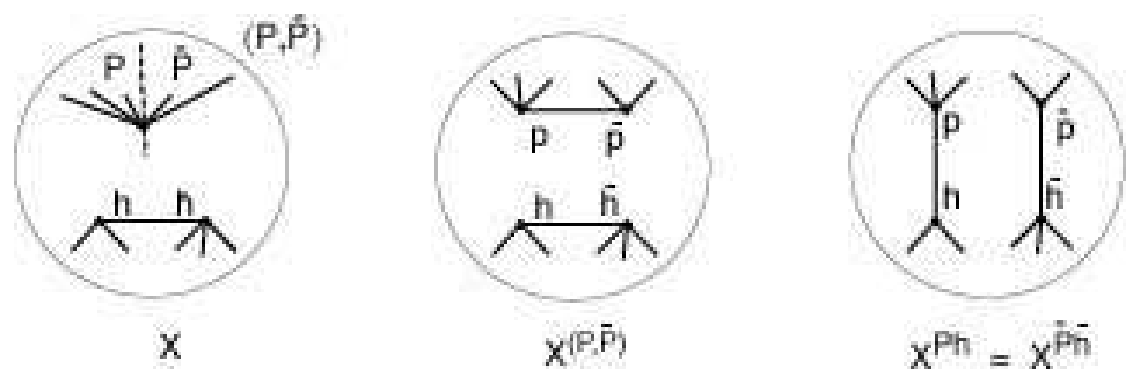

FIgURE 10. Decontracting a partition and a half-edge

Definition 5.5. $\delta_{H}: \mathcal{G}_{v} \rightarrow \mathcal{G}_{v+1}$ is defined by

$$
\delta_{H}(X)=\frac{1}{2} \sum_{P, h} X^{P h}
$$

where $h$ runs over all half edges of $X$, and $P$ over all subsets of the edges at all vertices.

The factor of $\frac{1}{2}$ is there to account for the fact that, since $X^{P h}=X^{\bar{P} \bar{h}}$, we have counted each graph in the coboundary twice.

Proposition 5.6. The following diagrams commute.

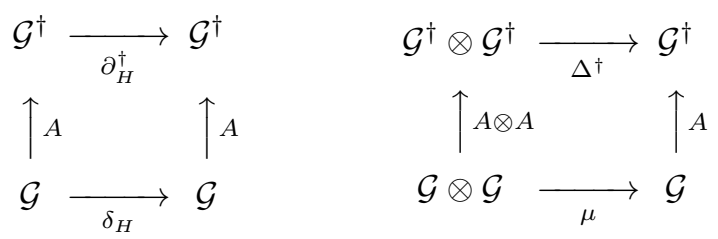


Proof. We first show that the left-hand diagram commutes.

Start in the lower-left with a graph $X$. Trace through the diagram in both ways, evaluating in the upper-right hand corner on the graph $Y$. Let $A_{+}$denote the set $\left\{(P, h): X^{P h}=Y\right\}$. Let $A_{-}$denote the set $\left\{(P, h): X^{P h}=-Y\right\}$. Going right and then up in our diagram, we get

$$
<\sum X^{P h}, Y>=\left|A u t\left(X^{P h}\right)\right|\left(\left|A_{+}\right|-\left|A_{-}\right|\right)=|A u t(Y)|\left(\left|A_{+}\right|-\left|A_{-}\right|\right) .
$$

Let $B_{+}$denote the set $\left\{h, k: Y_{h k}=X\right\}$. Let $B_{-}$denote the set $\left\{h, k: Y_{h k}=-X\right\}$. Going up and then right in the diagram:

$$
<X, \partial_{H}(Y)>=|\operatorname{Aut}(X)|\left(\left|B_{+}\right|-\left|B_{-}\right|\right) .
$$

Thus it suffices to show that $|\operatorname{Aut}(Y)|\left|A_{ \pm}\right|=|\operatorname{Aut}(X)|\left|B_{ \pm}\right|$. Now $\operatorname{Aut}(X)$ acts transitively on each of $A_{ \pm}$and $\operatorname{Aut}(Y)$ acts transitively on each of $B_{ \pm}$, since we may assume that neither $X$ nor $Y$ have orientation reversing automorphisms. Thus $|\operatorname{Aut}(X)| /\left|\operatorname{stab}\left(a_{ \pm}\right)\right|=\left|A_{ \pm}\right|$and $|\operatorname{Aut}(Y)| /\left|\operatorname{stab}\left(b_{ \pm}\right)\right|=\left|B_{ \pm}\right|$, where $\operatorname{stab}\left(a_{ \pm}\right)$, $\operatorname{stab}\left(b_{ \pm}\right)$denote the stabilizers of the elements $a_{ \pm} \in A_{ \pm}$and $b_{ \pm} \in B_{ \pm}$respectively. It is thus sufficient to show that $\left|\operatorname{stab}\left(a_{ \pm}\right)\right|=\left|\operatorname{stab}\left(b_{ \pm}\right)\right|$. To see this, suppose that $a_{ \pm}=(P, h)$. Notice that every automorphism of $X$ which fixes $P$ and $h$ defines an automorphism of $Y= \pm X^{P h}$. Similarly every automorphism of $Y$ which fixes $\{h, k\}$ extends to an automorphism of $X= \pm Y_{h k}$. This gives us inverse maps between $\operatorname{stab}\left(a_{ \pm}\right)$and $\operatorname{stab}\left(b_{ \pm}\right)$.

Now we turn to the second commutative diagram. Start in the lower left with a tensor

$$
X_{1}^{n_{1}} \cdots X_{p}^{n_{p}} \otimes X_{1}^{m_{1}} \cdots X_{p}^{m_{p}}
$$

where each $X_{i}$ is a connected graph and $m_{i}, n_{i} \geq 0$. To establish commutativity, it suffices to evaluate in the upper right on the graph

$$
X_{1}^{m_{1}+n_{1}} \cdots X_{k}^{m_{k}+n_{k}},
$$

since evaluating on other monomials is zero in both directions. We may also assume that either $X_{i}$ has an even number of vertices, or that $m_{i}+n_{i}=1$, since otherwise the oriented graph itself is zero. Therefore we may suppose that the tensor in the lower-left corner is of the form

$$
X_{1}^{n_{1}} \cdots X_{k}^{n_{k}} Y_{1} \cdots Y_{s} \otimes X_{1}^{m_{1}} \cdots X_{k}^{m_{k}} Y_{s+1} \cdots Y_{t}
$$

where $X_{i}$ are distinct even graphs, and the $Y_{i}$ are distinct odd graphs. We evaluate in the upper right on the monomial $X_{1}^{m_{1}+n_{1}} \cdots X_{k}^{m_{k}+n_{k}} Y_{1} \cdots Y_{t}$. To do this, when we go up and then right, we must calculate

$$
\begin{aligned}
& \Delta\left(X_{1}^{m_{1}+n_{1}} \cdots X_{k}^{m_{k}+n_{k}} Y_{1} \cdots Y_{t}\right)=\ldots+ \\
& \left(\begin{array}{c}
m_{1}+n_{1} \\
m_{1}
\end{array}\right) \cdots\left(\begin{array}{c}
m_{k}+n_{k} \\
m_{k}
\end{array}\right) X_{1}^{n_{1}} \cdots X_{k}^{n_{k}} Y_{1} \cdots Y_{s} \otimes X_{1}^{m_{1}} \ldots X_{k}^{m_{k}} Y_{s+1} \cdots Y_{t} \\
& +\cdots
\end{aligned}
$$

Hence, going up and right we get

$$
\begin{aligned}
& \left|A u t\left(X_{1}^{n_{1}} \cdots X_{k}^{n_{k}} Y_{1} \cdots Y_{s}\right)\right| \cdot \\
& \qquad\left|\operatorname{Aut}\left(X_{1}^{m_{1}} \cdots X_{k}^{m_{k}} Y_{s+1} \cdots Y_{t}\right)\right| \cdot\left(\begin{array}{c}
m_{1}+n_{1} \\
m_{1}
\end{array}\right) \cdots\left(\begin{array}{c}
m_{k}+n_{k} \\
m_{k}
\end{array}\right) .
\end{aligned}
$$


On the other hand, going right and then up, we get simply

$$
\left|A u t\left(X_{1}^{m_{1}+n_{1}} \cdots X_{k}^{m_{k}+n_{k}} Y_{1} \cdots Y_{t}\right)\right| \text {. }
$$

Equality follows from the fact that for any graph $X,\left|A u t\left(X^{p+q}\right)\right|=\left|\operatorname{Aut}\left(X^{p}\right)\right|\left|A u t\left(X^{q}\right)\right|\left(\begin{array}{c}p+q \\ p\end{array}\right)$; this is a consequence of the formula $\left|A u t\left(X^{n}\right)\right|=n !|A u t(X)|^{n}$, coming from the fact that $\operatorname{Aut}\left(X^{n}\right)$ is the semidirect product of the symmetric group $\Sigma_{n}$ and $\operatorname{Aut}(X)^{n}$.

Proposition 5.3 Note that by definition, $\theta=\Delta \partial_{H}-\partial_{H} \Delta=\Delta \partial_{H}-\left(\partial_{H} \otimes i d+\right.$ $\left.\tau\left(\partial_{H} \otimes i d\right) \tau\right) \Delta$, and therefore $\theta^{\dagger}=\partial_{H}^{\dagger} \Delta^{\dagger}-\Delta^{\dagger}\left(\partial_{H}^{\dagger} \otimes i d+\tau\left(\partial_{H}^{\dagger} \otimes i d\right) \tau\right)$. Hence $\hat{\theta}=A^{-1} \theta^{\dagger}(A \otimes A)=\delta_{H} \mu-\mu\left(\delta_{H} \otimes i d+\tau\left(\delta_{H} \otimes i d\right) \tau\right)$ is the deviation from $\delta_{H}$ being a derivation. Now by the first commutative diagram in the previous proposition, $\delta_{H}^{2}=0$. Also, $\delta_{H}$ satisfies the BV axiom, since it is of degree 1 and involves summing over pairs of sub-objects of graphs (just like $\partial_{H}$ ). Therefore by Proposition 4.8 this deviation satisfies the graded Jacobi identity:

$$
\hat{\theta} \circ(\hat{\theta} \otimes i d) \circ\left(i d+\sigma+\sigma^{2}\right)=0 .
$$

This implies that $\theta^{\dagger}$ satisfies the graded Jacobi identity by the following commutative diagram:

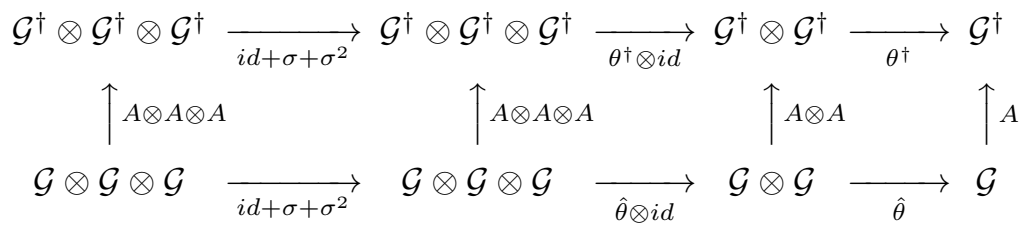

Now dualizing, $\theta$ satisfies graded co-Jacobi, which implies that $\theta^{\phi}$ is a graded Lie cobracket.

\section{Compatibility of BRACKet AND COBRACKET}

Recall that if the bracket $b=[\cdot, \cdot]$ and cobracket $\theta$ satisfy the compatibility relation

$$
\theta[v, w]+[\theta(v), w]+(-1)^{|\theta||v|}[v, \theta(w)]=0,
$$

then the shifted complex $\mathcal{G}[-1]$ is a graded Lie bialgebra.

In our case, the cobracket $\theta$ is of degree -1 (though $\theta^{\phi}$ is of degree 0 ). The graph complex $\mathcal{G}$ does not satisfy the compatibility relation, as one can verify by computing using the graphs $X$ and $Y$ depicted in Figure 11 The problem in this example is that both graphs $X$ and $Y$ have separating edges, so that some terms in the bracket $[X, Y]$ are not connected. To remedy this, we consider the subspace $\mathcal{H} \subset \mathcal{G}$ generated by connected graphs with no separating edges. In the literature these are often called one-particle irreducible graphs. Note that $\mathcal{H}$ is a subcomplex of $\mathcal{G}$ with respect to the boundary operator $\partial_{E}$, though it is not with respect to $\partial_{H}$.

The following two lemmas are easily verified:

Lemma 6.1. If $X$ and $Y$ are connected graphs, and $X$ has no separating edges, then each term $(X \cdot Y)_{h k}$ in the bracket $[X, Y]$ is connected. If in addition $Y$ has no separating edges, then each term has no separating edges.

Lemma 6.2. If $X$ has no separating edges, and $X_{h k}$ has two components, then each component has no separating edges. 

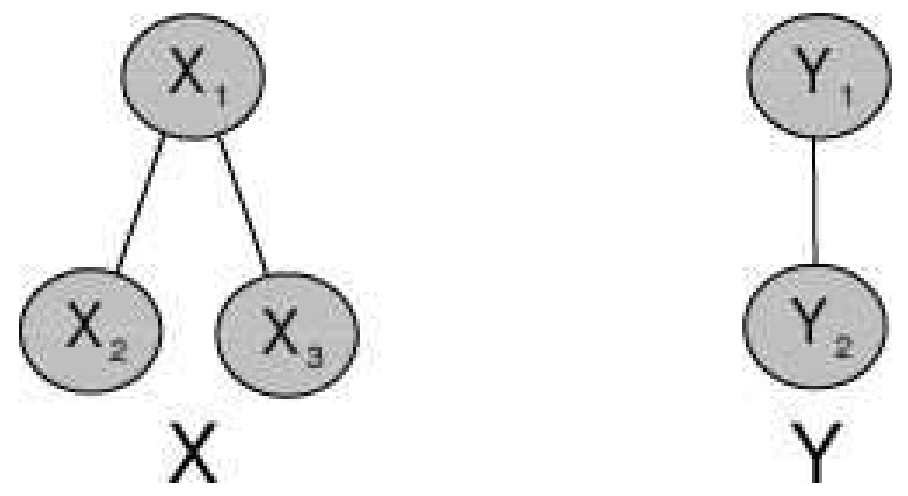

Figure 11. Bracket and cobracket not compatible

Theorem 6.3. The subcomplex $\mathcal{H} \subset \mathcal{G}$ spanned by one-particle irreducible graphs is a graded Lie bialgebra with respect to $b^{\phi}=[\cdot, \cdot]^{\phi}$ and $\theta^{\phi}$.

Proof. The Lemmas show that bracket and cobracket restrict to operations on $\mathcal{H}$. To prove the compatibility relation, we will show that all terms in the sum $\theta[X, Y]+[\theta(X), Y]+(-1)^{x}[X, \theta(Y)]$ cancel in pairs. As usual, $x$ denotes the number of vertices of $X$.

Because $\mathcal{H}$ has a basis consisting of connected graphs, multiplication actually induces an isomporphism $\mu: \mathcal{H} \odot \mathcal{H} \rightarrow \mathcal{H}^{2}$. Composing $\mu$ with $\theta$ simplifies the expression for the cobracket to:

$$
\mu \theta(X)=\sum_{\{h, k\} \text { separating }} X_{h k} .
$$

We also have $\mu[X \odot Y, Z]=[X Y, Z]$ and $\mu[X, Y \odot Z]=[X, Y Z]$, since the bracket satisfies the Gerstenhaber axiom, so that showing that $\theta[X, Y]+[\theta(X), Y]+(-1)^{x}[X, \theta(Y)]$ vanishes is equivalent to showing that

$$
\mu \theta[X, Y]+[\mu \theta(X), Y]+(-1)^{x}[X, \mu \theta(Y)]=0 .
$$

We have

$$
\mu \theta[X, Y]=\mu \theta\left(\sum_{h \in X, k \in Y}(X \cdot Y)_{h k}\right)=\sum_{h \in X, k \in Y} \sum_{r, s}\left((X \cdot Y)_{h k}\right)_{r s},
$$

where $r, s$ are separating half edges in $(X \cdot Y)_{h k}$.

For each term $\left((X \cdot Y)_{h k}\right)_{r s}$ of $\mu \theta[X, Y]$ there are several cases.

Case 1. $r, s \subset X, r, s \neq \bar{h}$.

If $\{r, s\}$ is not a separating pair in $X$, then the edge $e(k)$ containing $k$ must be a separating edge in $Y$, contradicting our assumptions. Thus $(X \cdot Y)\langle h k\rangle$ are arranged as in Figure 12 where $e(r)$ contains $r$ and $e(s)$ contains $s$.

Then $X_{r s}$ is a term of $\mu \theta(X)$, and $\left(X_{r s} Y\right)_{h k}=\left((X Y)_{r s}\right)_{h k}$ is a term of $[\mu \theta(X), Y]$, which cancels with $\left((X Y)_{h k}\right)_{r s}$ by Lemma 2.4

Case 2. $r, s \subset Y, r, s \neq \bar{k}$.

This is similar to the last case (see Figure 13). $Y_{r s}$ is a term of $\mu \theta(Y)$ and $\left(X \cdot Y_{r s}\right)_{h k}=(-1)^{x}\left((X Y)_{r s}\right)_{h k}$ is a term of $(-1)^{x}[X, \mu \theta(Y)]$ which cancels with $\left((X Y)_{h k}\right)_{r s}$.

Case 3. $r \subset X, s \subset Y, r \neq \bar{h}, s \neq \bar{k}$ 


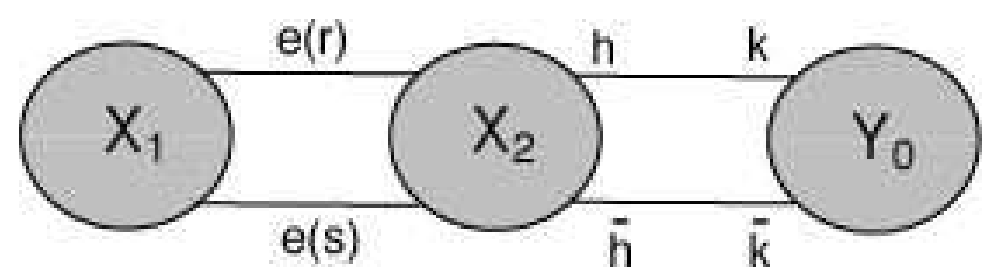

Figure 12. $(X \cdot Y)\langle h k\rangle$ in Case $1(r, s \subset X, r, s \neq \bar{h})$

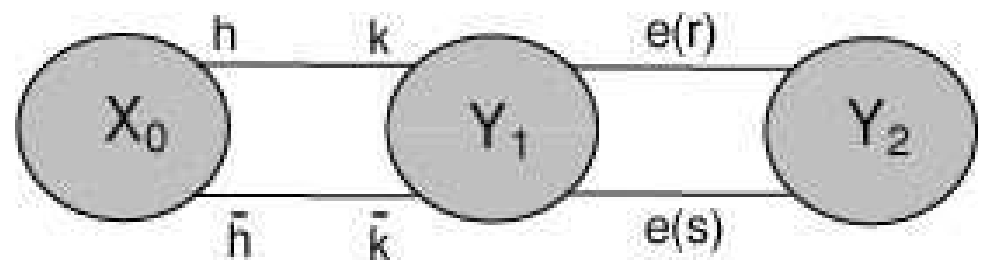

Figure 13. $(X \cdot Y)\langle h k\rangle$ in Case $2(r, s \subset Y, r, s \neq \bar{k})$

Here $(X \cdot Y)\langle h k\rangle$ must be in the configuration of Figure 14 since neither $X$ nor $Y$ has a separating edge.

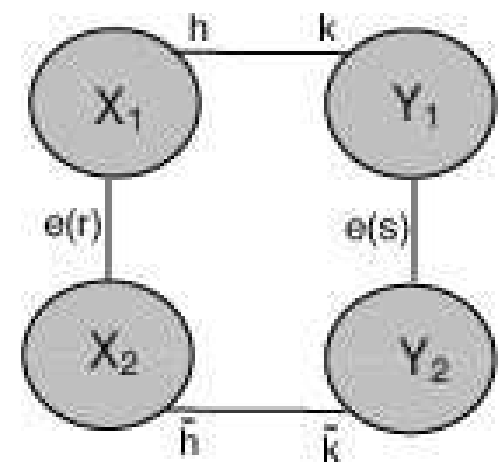

Figure 14. $(X \cdot Y)\langle h k\rangle$ in Case $3(r \subset X, s \subset Y, r \neq \bar{h}, s \neq \bar{k})$

But then $(X Y)_{r s}$ is a term of $[X, Y]$ and $\left((X Y)_{r s}\right)_{h k}$ is a term of $\mu \theta[X, Y]$ which cancels with $\left((X Y)_{h k}\right)_{r s}$.

Case 4. $r=\bar{h}$

$(X \cdot Y)\langle h k\rangle$ must be as in Figure 15.

In this case $X_{r s}$ is a term of $\mu \theta(X)$, and so $\left(X_{r s}\right)_{h k}$ is a term of $[\mu \theta(X), Y]$ which cancels.

Case 5. $r=\bar{k}$

This is similar to case 4 (see Figure 16

Here $(-1)^{x}\left((X Y)_{r s}\right)_{h k}=\left(X \cdot Y_{r s}\right)_{h k}$ is a term of $(-1)^{x}[X, \mu \theta(Y)]$ which cancels. 


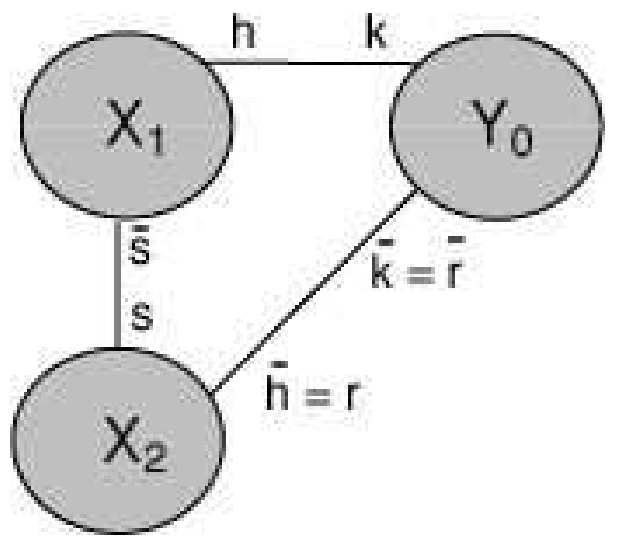

Figure 15. $(X \cdot Y)\langle h k\rangle$ in Case $4(r=\bar{h})$

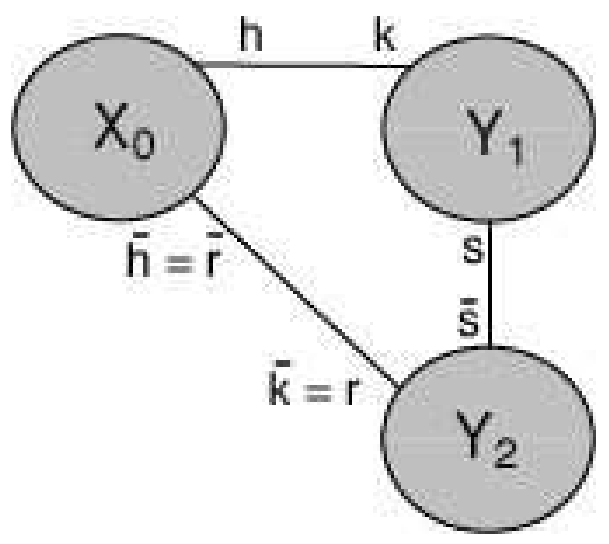

FiguRe 16. $(X \cdot Y)\langle h k\rangle$ in Case $6(r=\bar{k})$

Thus we have shown that each term of $\mu \theta[X, Y]$ cancels either with another term of $\mu \theta[X, Y]$ or with a term of $[\mu \theta(X), Y]$ or $(-1)^{h}[X, \mu \theta(Y)]$. Also, each term of $[\mu \theta(X), Y]$ and $(-1)^{x}[X, \mu \theta(Y)]$ does, in fact, cancel with a term in $\mu \theta[X, Y]$.

Remark: The technique of this proof can be used to give an easy argument that $\theta^{2}=0$ on $\mathcal{H}$.

The fact that the subcomplex $\mathcal{H}$ carries a bi-algebra structure is valid in the general setting of cyclic operads. For the associative and Lie cases, $\mathcal{H}$ is quasiisomorphic to $\operatorname{Prim}(\mathcal{G})$ (see $[\mathrm{CoV}]$ ), so that we have a bi-algebra structure on chain complexes $\mathcal{H}$ which compute the cohomology of mapping class groups (associative operad) and of groups of outer automorphism groups of free groups (Lie operad). In the commutative case, which we have focused on in this paper, computer calculations due to F. Gerlits Gts show that the map $H_{*}(\mathcal{H}) \rightarrow H_{*}(\operatorname{Prim}(\mathcal{G}))$ is not surjective. However, in [CoGV] we show that the Lie bracket and cobracket described in this paper do induce a Lie bi-algebra structure on a certain quotient 
complex $\operatorname{Prim}(\mathcal{G}) / \mathcal{C}$ which is quasi-isomorphic to $\operatorname{Prim}(\mathcal{G})$, so in this case as well we have a bi-algebra structure on a chain complex which computes graph homology.

\section{HomOLOGY}

In this section we consider $\mathcal{G}$ as a complex with boundary operator $\partial_{E}$.

Proposition 7.1. The graph bracket descends to the level of homology.

Proof. This follows from the identity

$$
\partial_{E}[X, Y]=\left[\partial_{E} X, Y\right]+(-1)^{x}\left[X, \partial_{E} Y\right]
$$

Which can be derived by expanding the identity

$$
\left(\partial_{H} \partial_{E}+\partial_{H} \partial_{E}\right)(X Y)=0 .
$$

Proposition 7.2. The graph cobracket descends to the level of homology.

Proof. The proof of the previous lemma can be dualized to yield a proof of the present lemma by considering the equation

$$
\Delta\left(\left(\partial_{H} \partial_{E}+\partial_{E} \partial_{H}\right) X\right)=0 .
$$

In the course of expanding this out one must use the fact that $\partial_{E}$ is a coderivation and that $\partial_{H} \otimes i d+i d \otimes \partial_{H}$ anti-commutes with $\partial_{E} \otimes i d+i d \otimes \partial_{E}$, where one must as always respect the Koszul rule of signs.

It turns out that the bracket can be interpreted in terms of the Lie algebra $c_{n}$ mentioned in the introduction. Kontsevich constructs an isomorphism between $\mathfrak{s p}(2 n)$-invariants in $\Lambda^{*} c_{n}$ and elements of $\mathcal{G}$. More specifically, there is a map

$$
\phi_{n}:\left(\Lambda c_{n}\right)^{\mathfrak{s p}(2 n)} \rightarrow \mathcal{G}
$$

See $\mathrm{CoV}$.

Proposition 7.3. $\phi_{n}$ is a Lie algebra homomorphism. That is, it maps the Schouten bracket to the graph bracket.

Proof. We recall that an $\mathfrak{s p}(2 n)$ invariant tensor is associated to a graph by the following procedure. Each vertex of the graph represents a tensor factor, in the order given by the vertex labelling. For each edge we put a $p_{i}$ at the tail of the arrow and a $q_{i}$ at the head or we put the $q_{i}$ at the tail and the $p_{i}$ at the head, incurring a minus sign as a result. We sum over all possible choices, each choice is called a "state." Passing to the wedge product yields an $\mathfrak{s p}(2 n)$-invariant. The Schouten bracket involves first choosing two tensor factors to bracket, which means picking a vertex from each of the two graphs. Then one takes the Poisson bracket of the monomials at each vertex. This can be thought of as deleting a $p_{i}$ from one and a $q_{i}$ from the other, and then multiplying the monomials together. One can view the result as breaking the edges with the $p_{i}$ and $q_{i}$ into half-edges, gluing them together and contracting, and also gluing together the resulting dangling edges. Summing over all possible states, we see that this contribution to the Schouten bracket is given by contracting the two given half-edges, which is the definition of the bracket. 
It is not difficult to show that the Schouten bracket is always trivial on the homology level: one can think of it as the deviation of the Chevalley-Eilenberg differential from being a derivation with respect to the wedge product. Therefore, the preceding proposition suggests that the bracket is trivial on the homology level. This is not quite true, however, since $\phi_{n}$ is not a chain map! (This was an oversight in Kontsevich's argument. In $\mathrm{CoV}]$ we show how to repair this oversight.) However, there is a straightforward proof of the homological triviality of the bracket, due to S. Mahajan.

Proposition 7.4. The bracket $[\cdot, \cdot]$ is trivial on the homology level.

Proof. Define a multiplication $\mu_{1}: \mathcal{G} \otimes \mathcal{G} \rightarrow \mathcal{G}$ by

$$
\mu_{1}(X \otimes Y)=\sum_{x \in X, y \in Y}(X \cdot Y)<x y>
$$

(See Figure 3) Then an easy argument shows that $[X, Y]=\partial_{E} \circ \mu_{1}-\mu_{1} \circ \partial_{E}$. Therefore, if $X$ and $Y$ are both cycles, so is $X \cdot Y$, and $[X, Y]=\partial_{E} \mu_{1}(X \otimes Y)$.

A similar argument shows

Proposition 7.5. The cobracket $\theta$ is trivial on the homology level.

Proof. This follows by a very similar argument to the previous proposition. Define $\Delta_{1}: \mathcal{G} \rightarrow \mathcal{G} \otimes \mathcal{G}$ in the same way as $\theta$ only don't contract an edge. Then $\theta=$ $\partial_{E} \circ \Delta_{1}-\Delta_{1} \circ \partial_{E}$.

Finally, we show that $\partial_{H}$ is zero at the homology level as well.

Proposition 7.6. $\partial_{H}$ is zero on homology.

Proof. Define a map $\alpha: \mathcal{G} \rightarrow \mathcal{G}$ as

$$
\alpha(X)=\frac{1}{2} \sum_{x, y, x \neq \bar{y}} X<x y>.
$$

It is straightforward that $\partial_{E} \alpha-\alpha \partial_{E}=\partial_{H}$.

\section{REFERENCES}

[BGRT] D. Bar-Natan, S. Garoufalidis, L. Rozansky and D. Thurston, The Aarhus integral of rational homology 3-spheres I: A highly nontrivial flat connection on $S^{3}$., to appear in Selecta Mathematica

[Co] J. Conant, Fusion and fission in graph complexes, to appear in Pacific Journal of Mathematics, see also math.QA/0208093

$[\mathrm{CoV}]$ J. Conant and K. Vogtmann, On a theorem of Kontsevich, math.QA/0208169

[CoGV] J. Conant, F. Gerlits and K. Vogtmann, Separating vertices in graphs, in preparation.

[CuV] Marc Culler and Karen Vogtmann, Moduli of graphs and automorphisms of free groups. Invent. Math. 84 (1986), no. 1, 91-119.

[CS] Moira Chas and Dennis Sullivan, String topology, preprint 1999, math.GT/9911159

[C] Moira Chas, Combinatorial Lie bialgebras of curves on surfaces, preprint 2001, math.GT/0105178

[GL] Stavros Garoufalidis and Jerome Levine, Tree level invariants of three-manifolds, massey products and the Johnson homomorphism, preprint 1999, math.GT/9904106

[GK] Ezra Getzler and M.M. Kapranov, Cyclic operads and cyclic homology Geometry, topology, and physics, 167-201, Conf. Proc. Lecture Notes Geom. Topology, IV, Internat. Press, Cambridge, MA, 1995. 
[GiK] V. Ginzburg and M. Kapranov, Koszul duality for operads, Duke Math. J. 76 (1994), no. $1,203-272$

[GK2] E. Getzler and M. M. Kapranov, Modular operads Compositio Math. 110 (1998), no. $1,65-126$.

[Gts] Ferenc Gerlits, Calculations in graph homology, in preparation.

[Ge] Ezra Getzler, Batalin-Vilkovisky algebras and two-dimensional topological field theories, preprint 2001.

[K1] Maxim Kontsevich, Formal (non)commutative symplectic geometry. The Gelfand Mathematical Seminars, 1990-1992, 173-187, Birkhuser Boston, Boston, MA, 1993.

[K2] Maxim Kontsevich, Feynman diagrams and low-dimensional topology. First European Congress of Mathematics, Vol. II (Paris, 1992), 97-121, Progr. Math., 120, Birkhuser, Basel, 1994.

[KT] Greg Kuperberg and Dylan P. Thurston Perturbative 3-manifold invariants by cutand-paste topology, preprint 1999, UC Davis Math 1999-36, math.GT/9912167

[LMO] T.Q.T. Le, J. Murakami and T. Ohtsuki, On a universal perturbative invariant of 3-manifolds, Topology 37-3 (1998)

[L] Jerome Levine, Homology cylinders, an expansion of the mapping class group, Algebr. Geom. Topol. 1(2001) 551-578

[M] Shahn Majid, Foundations of quantum group theory. Cambridge University Press, Cambridge, 1995. x+607 pp.

[Ml] Martin Markl, Cyclic operads and homology of graph complexes, Rendiconti del circolo matematico di Palermo, serie II, Suppl. 59 (1999) pp.161-170

[M-M] John W. Milnor and John C. Moore, On the structure of Hopf algebras. Ann. of Math. (2) 811965 211-264.

[P] R. C. Penner,Perturbative series and the moduli space of Riemann surfaces. J. Differential Geom. 27 (1988), no. 1, 35-53.

[T] Dylan Thurston, Undergraduate thesis, Harvard.

Cornell University

Cornell University 